\title{
Heart failure with preserved left ventricular ejection fraction. Revision of exceptional case of valvular and subvalvular aortic obstruction
}

\author{
Diego A Valadez-Villegas ${ }^{1}$, Guillermo Trujillo-Martínez ${ }^{1}$, Pamela Morales-Álvarez ${ }^{1}$, Roberto Cano-Zarate ${ }^{2}$, Luis Antonio Ferraez-Servin \\ de la Mora ${ }^{1}$, Erick Alexanderson-Rosas ${ }^{1,3}$ and Nilda Espinola-Zavaleta ${ }^{1,4 *}$ \\ ${ }^{1}$ Department of Nuclear Cardiology, National Institute of Cardiology Ignacio Chávez, Mexico City, México \\ ${ }^{2}$ Department of Magnetic Resonance, National Institute of Cardiology Ignacio Chávez, Mexico City, México \\ ${ }^{3}$ Faculty of Medicine, National Autonomous University of Mexico City, México \\ ${ }^{4}$ Department of Echocardiography, ABC Medical Center, Mexico City, Mexico
}

\begin{abstract}
Subvalvular stenosis represents the second most common cause of aortic stenosis. However, it remains a rare disease. Its importance relays in a possible development of heart failure at an early age and the association with multiple cardiac defects. Surgical treatment is definitive resolution.

In this case report, we describe a 28 years old man who begins with progressive dyspnea and palpitations since he was 17 years old. Later, the patients required a double valvular replacement due to a complex valvular and subvalvular aortic lesion that was diagnosed by echocardiography and cardiac magnetic resonance.

Echocardiography and cardiac magnetic resonance are the diagnostic standard for this particular condition since these techniques allow characterizing the lesion and its hemodynamic repercussion in cardiac cavities and establishing the treatment.
\end{abstract}

\section{Introduction}

Fixed aortic subvalvular stenosis is a rare congenital anomaly that represents $1 \%$ of all congenital heart defects [1]. Fixed aortic subvalvular stenosis may be due to a discrete fibrous membrane, muscle narrowing or a combination of both [1]. The obstruction may be focal, as in a discrete membrane, or a more diffuse tunnel type; the latter is associated with a greater degree of stenosis. Rarely, the tissue or cords of the accessory mitral valve can cause fixed aortic subvalvular stenosis.

Rosenquist, et al. found that the distance between the mitral and aortic valves in patients with aortic subvalvular stenosis is constantly increasing. They hypothesized that alteration in the direction of blood flow near the crest of the interventricular septum leads to the differentiation of embryonic cells in a variant of fibrotic tissue [2]. The bicuspid aortic valve may be present in $23 \%$ of these patients. Subaortic stenosis can also occur as part of the Shone complex, which often includes the parachute mitral valve with mitral stenosis, bicuspid aorta and coarctation of the aorta. Nearly $37 \%$ of patients may also have concomitant perimembranous ventricular septal defects. These defects usually affect men more often than women with a 2:1 ratio [3].

The 2017 ESC guidelines consider echocardiography the gold standard to diagnose and characterize these subvalvular and valvular obstructive congenital anomalies. They also evaluate the integrity of the aortic and mitral valves and assess the hemodynamic and functional impact on the cardiovascular system including the fibrosis pattern $[1,4]$. However, magnetic resonance imaging may offer complementary information in patients with discrepant or inadequate quality echocardiographic results [4].
This report describes the case of a male patient in the third decade of life, who in his late adolescence, showed progressive exertional dyspnea associated with frequent paroxysmal palpitations. Echocardiography and magnetic resonance imaging evidenced three rare causes of subvalvular and aortic valve obstruction.

\section{Case presentation}

A 28-year-old man was referred to our institution for presenting dyspnea on great efforts since he was 17 years old, which progressed to medium efforts in the last 6 months. On the physical examination, he was hemodynamically stable with vital signs within normal parameters. He had rhythmic carotid pulses with a slow, vibrated ascent and a diminished amplitude (parvus et tardus). A single S1 heart sound, a diffused S2 heart sound and a grade III/VI aortic ejection murmur radiating to suprasternal neck and a long, diastolic aortic murmur were heard. Normal breathing noises, without evidence of visceromegaly or edema of the pelvic limbs. The electrocardiogram showed a sinus rhythm with heart rate of $100 \mathrm{bpm}$, left atrial enlargement and left bundle branch block (Figure 1). Laboratory studies were found within normal parameters. Chest $\mathrm{x}$-ray showed grade II cardiomegaly,

${ }^{\star}$ Correspondence to: Nilda Espinola-Zavaleta, $\mathrm{MD}, \mathrm{PhD}$, Department of Nuclear Cardiology, Juan Badiano № 1, Colonia Sección XVI, PC 14080, Tlalpan, Mexico City, Mexico, E-mail: niesza2001@hotmail.com

Key words: mitral accessory tissue, subaortic stenosis, bicuspid aortic valve, subaortic ridge, echocardiography, cardiac magnetic resonance

Received: March 15, 2020; Accepted: April 02, 2020; Published: April 07, 2020 
without pulmonary congestion. Transthoracic and transesophageal echocardiography demonstrated:

- A bicuspid aortic valve type 3 with fusion of the non-coronary leaflet and right coronary leaflet

- A subvalvular stenosis due to subaortic fibrous ridge and accessory mitral tissue (Figures 2 and 3) with a maximum velocity of $5.1 \mathrm{~m} / \mathrm{s}$, a maximum gradient $104 \mathrm{mmHg}$ and average gradient of $55 \mathrm{mmHg}$

- Significant aortic insufficiency with vena contracta of $7.5 \mathrm{~mm}$ (Figure 3)

- Moderate mitral regurgitation (Figure 3)

- A permeable oval foramen (Figure 2)

On the other hand, the echocardiography also showed severe asymmetric septal hypertrophy with a basal thickness of $22 \mathrm{~mm}$, relative parietal thickness of 1.49 and left ventricular mass index of $199 \mathrm{gr} / \mathrm{m}^{2}$, left ventricular ejection fraction of 70\%, type I diastolic dysfunction and normal pulmonary artery systolic pressure.

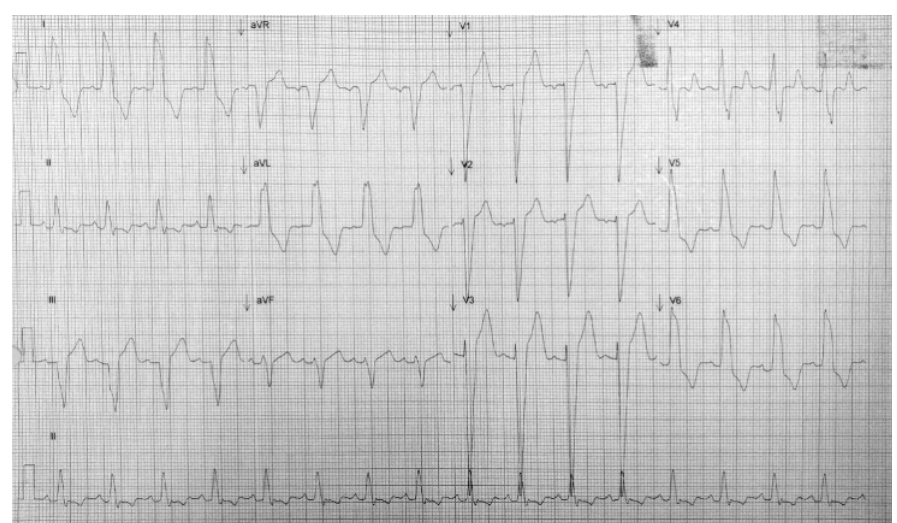

Figure 1. 12-lead resting electrocardiogram in sinus rhythm with heart rate of $100 \mathrm{bpm}$, left atrial enlargement and left bundle branch block

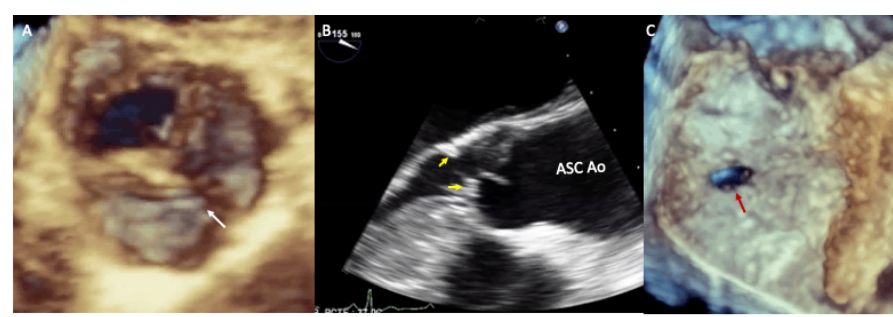

Figure 2. Two-dimensional and three-dimensional transesophageal echocardiogram showing: A-bicuspid aortic valve with raphe (white arrow), B- Zoom-in of the left ventricular outflow tract with subvalvular ridge (yellow arrowheads), aortic valve and ascending aorta, C- Patent oval foramen (red arrow). Abbreviations: Asc Ao-ascending aorta

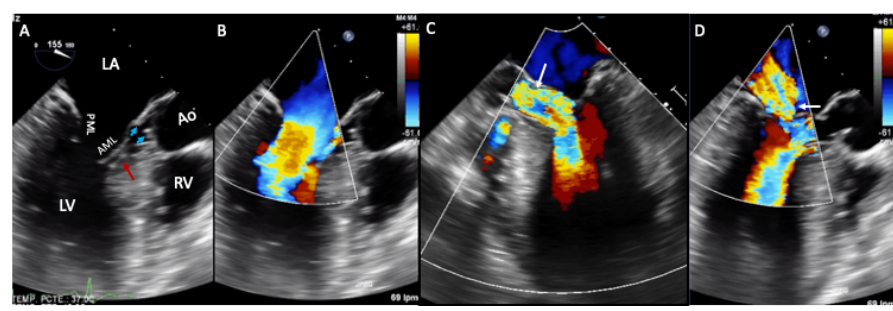

Figure 3. Two-dimensional transesophageal echocardiogram with color Doppler. A, BImages at $155^{\circ}$ showing left atrial dilation, accessory mitral tissue (red arrow), subaortic ridge (blue arrowheads) and septal hypertrophy, C- Severe aortic regurgitation, (white arrow) D-Moderate mitral regurgitation, (white arrow). Abbreviations: LA-left atrium; LV-left ventricle; RV-right ventricle; AML: anterior mitral leaflet; PML: posterior mitral leaflet; Ao-Aorta

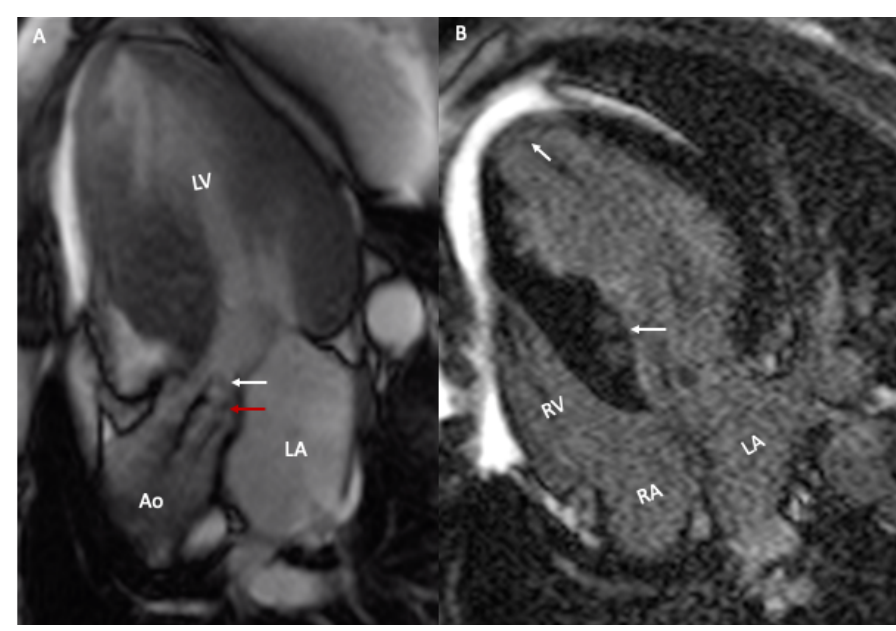

Figure 4. Magnetic resonance imaging. A- Gradient echo cine in 3 chambers, subvalvular acceleration (white arrow) and aortic valve (red arrow) and asymmetric septal hypertrophy are observed. B- In the recovery inversion sequence, late patchy intramyocardial enhancement and diffuse septal and left ventricular apex enhancement are observed (arrows)

Magnetic resonance imaging corroborated the aortic subvalvular and valvular obstruction and demonstrated septal and apical fibrosis (Figure 4). Cardiac catheterization reported coronary arteries without obstructive lesions. The patient underwent surgery for aortic and mitral valve replacement, resection of the subaortic fibrous ridge and a Morrow septal myomectomy. Currently, the patient is in NYHA I functional class, with periodic follow-up in the outpatient clinic.

\section{Discussion}

Aortic stenosis is the most common valvulopathy in the world. Its importance relies in the poor prognosis from the onset of symptoms, reaching a mortality of up to $25 \%$ per year [5]. In this patient, three congenital anomalies causing aortic obstruction can be distinguished: bicuspid aortic valve, subaortic fibromuscular ridge and accessory mitral tissue or trileaflet mitral valve.

The prevalence of the bicuspid aortic valve is of $0.5-1.4 \%$ in the general population [6]. The vast majority of patients that do not develop a maximum transaortic gradient greater than $40 \mathrm{mmHg}$ before the age of 20 , will not present hemodynamic alterations before the age of 70 [7]. However, when this defect is severe or associated with another malformation, the symptoms may appear from earlier ages. Up to $20 \%$ of subvalvular stenosis are associated with bicuspid aortic valve [8]. The most common type of bicuspid aortic valve is the type 1 , where there is a fusion of the right and left coronary leaflets; it occurs in $80 \%$ of cases [9]. Our patient presented a bicuspid aortic valve type 3 , with fusion of the non-coronary leaflet and the right coronary leaflet; This type is the rarest and represents only $0.5 \%$ of the bicuspid aortic valves. The stenotic variant of the bicuspid aortic valve causes chronic trauma from turbulent blood flow in the left ventricular outflow tract that causes fibrosis in the lower portion of the leaflets.

About $6 \%$ of patients with bicuspid aortic valve have abnormalities in the mitral valve. The most common is the parachute mitral valve [6]. Accessory mitral tissue is usually an extremely rare echocardiographic finding with a prevalence of $0.7 \%$; in most cases, it has no hemodynamic repercussions [6]. The embryological mechanism of accessory mitral tissue is not well described, but it is believed to be the result of abnormal separation of the mitral valve and endocardial cushions. The importance of accessory mitral tissue lies in its frequent association with interventricular septal defects, that reaches up to $65 \%$ 
of cases. In addition, its association with subaortic membranes or ridges is not uncommon, as occurred in our patient [10]. In fact, it is calculated that about $85 \%$ of accessory mitral tissue cases are associated with obstruction of the left ventricular outflow tract by two main mechanisms: the first because it exerts a mass effect and the second because it produces a deposit of fibrous tissue by the turbulent flow generation [11]. When the accessory mitral tissue produces subvalvular obstruction, the main symptom is dyspnea in $30 \%$ of the patients, although $40 \%$ remain asymptomatic [11].

Subvalvular aortic stenosis can produce septal hypertrophy in up to $75 \%$ of cases, as we observed in our patient [12]. Septal hypertrophy generates a subaortic dynamic obstruction as described in asymmetric septal hypertrophic cardiomyopathy.

Doppler echocardiography plays a very important role in the evaluation of these patients, since it determines the severity of the stenosis, assesses the associated malformations and guides the treatment. Three-dimensional echocardiography allows aortic valve and subvalvular obstruction to be characterized more precisely. In patients with subvalvular ridges, echocardiographic monitoring is essential, because they recurrence rate of about $30 \%$ and $15-20 \%$ require surgical reintervention $[10,13]$.

Magnetic resonance imaging can offer additional information to echocardiography. On the one hand, it offers an excellent characterization of myocardial tissue, unlimited windows and is considered the best non-invasive technique for measuring left ventricle volumes, mass and function [4]. Therefore, if a patient with aortic stenosis lacks information on his/her echocardiographic studies, magnetic resonance imaging can provide key data for the the diagnosis and prognosis of this disease, including the presence of fibrosis [4].

In the literature, it has been described that patients who develop a maximum gradient of the left ventricular outflow tract greater than $50 \mathrm{mmHg}$ should be operated [10]. Other indications for surgery are obstructive ventricular hypertrophy and clinically significant aortic regurgitation. The survival of these patients is $99 \%$ at 10 years and $86 \%$ at 20 years [10].

This case is of great clinical and echocardiographic interest because it is a patient who developed symptoms of heart failure with preserved ejection fraction, secondary to a complex obstruction that begins in the aortic valve and affects the left ventricular outflow tract with infrequent cardiac abnormalities [14-16].

\section{Conclusion}

The greatest implication of the subaortic ridge or accessory mitral tissue lies in their high frequency of association with other congenital malformations. Thus, although each of the elements of the obstruction of the left ventricular outflow tract are rare findings, they are statistically associated with each other. This report presents three extremely exceptional causes of aortic obstruction: type 3 bicuspid aortic valve, subaortic fibromuscular ridge, accessory mitral tissue and we could consider a fourth taking account asymmetric asymmetric septal hypertrophy, which is probably the result of the aforementioned defects.

Echocardiography and magnetic resonance imaging are the best methods to determine the association or presence of other heart defects and the hemodynamic repercussion.

\section{Highlights}

1. The alterations in the aortic valve are associated with subvalvular obstructions and abnormalities in the mitral valve.
2. There is a close relationship between the bicuspid aortic valve, subaortic fibromuscular ridges and interventricular septal hypertrophy.

3. The accessory mitral tissue is usually an asymptomatic finding, but occasionally it can cause obstruction of the left ventricular outflow tract.

4. The echocardiogram is the method of choice for the diagnosis and follow-up of patients with aortic and subaortic obstructive lesions. On the other hand, magnetic resonance imaging may provide additional information useful for the characterization of aortic stenosis and ventricular fibrosis.

\section{Acknowledgments}

None.

\section{Funding}

The present investigation has not received specific aid from public sector agencies, commercial sector or non-profit entities.

\section{Conflict of interest}

None.

\section{References}

1. Mulla S, Siddiqui WJ (2020) Subaortic Stenosis.

2. Rosenquist G, Clark E, McAllister H, Bharti S, Edwards J (1979) Increased MitralAortic Separation in Discrete Subaortic Stenosis. Circulation 60: 70-74.

3. Aboulhosn J, Child J (2006) Left Ventricular Outflow Obstruction: Subaortic Stenosis, Bicuspid Aortic Valve, Supravalvar Aortic Stenosis, and Coarctation of the Aorta. Circulation 114: 2412-2422.

4. Singh A, McCann GP (2019) Cardiac magnetic resonance imaging for the assessment of aortic stenosis. Heart 105: 489-497.

5. Ren X (2019) Aortic Stenosis. MedScape.

6. Van Rensburg A, Herbst P, Doubell A (2017) A retrospective analysis of mitral valve pathology in the setting of bicuspid aortic valves. Echo Res Prac 4: 21-28.

7. Ward C (2000) Clinical significance of the bicuspid aortic valve. Heart 83: 81-85.

8. Hauser M (2014) Fixed Subaortic Stenosis. Orphanet pp: 1-3.

9. Shabana A (2014) Bicuspid aortic valve. e-J Cardiol Prac 13: 2.

10. Brown D (2019) Subvalvar aortic stenosis (subaortic stenosis).

11. Manganaro R, Zito C, Khandheria $\mathrm{B}$, Cusmà-Piccione $\mathrm{M}$, Chiara Todaro $\mathrm{M}$, et al (2013) Accessory mitral valve tissue: an updated review of the literature. Eur Heart J-Cardiovasc Imaging 15: 489-497.

12. Brown JW1, Stevens LS, Holly S, Robison R, Rodefeld M, et al. (1988) Surgical spectrum of aortic stenosis in children: a thirty-year experience with 257 children. Ann Thorac Surg 45: 393-403. [Crossref]

13. Dodge-Khatami A, Schmid M, Rousson V, Fasnacht M, Doell C, et al. (2008) Risk factors for reoperation after relief of congenital subaortic stenosis. Eur J Cardiothorac Surg 8: 885-889.

14. Mordi I, Tzemos N (2012) Bicuspid Aortic Valve Disease: A Comprehensive Review. Cardiol Res Prac pp: 1-7.

15. Rabischoffsky A, de Freitas Portela AC, Magalhães M, Rabischoffsky R, Zanconato J, et al. (2018) Accessory Mitral Valve Tissue: An Unusual Echocardiographic Finding. CASE 2: 9-11.

16. Vilcant V, Hai O (2019) Left Ventricular Outflow Tract Obstruction.

Copyright: (C2020 Valadez-Villegas DA. This is an open-access article distributed under the terms of the Creative Commons Attribution License, which permits unrestricted use, distribution, and reproduction in any medium, provided the original author and source are credited. 